\title{
Urban sustainable development using qualimetry procedures of digital transformation
}

\author{
Dmitry Voronin*, Pavel Kuznetsov, and Vladislav Evstigneev \\ Sevastopol State University, Universitetskaya str., 33, 299053 Sevastopol, Russia
}

\begin{abstract}
The use of information technologies to improve the efficiency of organizing the functioning of urban processes and the provision of innovative services forms the basis of the concept of smart sustainable city. Society mistakenly identify the digital transformation of the urban environment with the unsystematic, redundant introduction of technical innovations into the citizens' life. This is because the changes made to the habitual processes of citizens' life often do not pass the mandatory check for their balance in terms of compliance with principles of sustainable development. The article proposes a new conceptual approach for assessing the effectiveness of the implemented solutions related to the implementation of the paradigm that ensures the transition from technology-oriented to human-oriented concept of "Sustainable Smart Cities". The main idea is to consider the transformation of the urban environment through the prism of changes in the functional state of its objects.
\end{abstract}

\section{Introduction}

Currently, one of the most common approaches to the development of urban infrastructure is the concept of "Smart City", which covers the vast majority of human life spheres: health care, security, transport, housing and communal services, education and tourism. In 2012, the United Nations Economic Commission for Europe formulated the concept of a "Smart Sustainable City" [1]. In 2015, indicators of the efficiency of its functioning were approved. However, many authors [2-5] still believe that the detailing of the proposed solutions is not sufficient when considering specific practical problems in this subject area. Moreover, now, the sustainable development of a smart urban environment by society is usually associated with a haphazard, excessive introduction of technical innovations into the life of citizens, disrupting the usual processes of their life. Any changes made to the urban environment should be verified for appropriateness in terms of various factors:

- minimization of conflict situations in society on the basis of hybrid simulation modeling and methods for solving multicriteria optimization problems;

- taking into account the interests of various social groups when using developed information technologies for big data analysis and agent systems simulation;

- compliance of the proposed technical and organizational solutions with the adopted city development strategy;

\footnotetext{
*Corresponding author: dima@voronins.com
} 
- ensuring the safety of the urban environment in accordance with the adopted regulatory documents;

- taking into account the basic components of the strategy of sustainable development of the city when making tactical decisions on the digital transformation of the urban environment [6-8].

Thus, a comfortable urban environment should be characterized by a set of contradictory criteria assessing the increase in the quality of human life for various social groups, and not be limited only to aspects of the implementation of information technologies [9-12]. These features necessitate the development of a new concept of the methodological approach, including the qualimetry procedures for the digital transformation of the urban environment, described in this work.

\section{Methods and types of the urban environment transformation}

In accordance with the principles of the systematic approach, the urban environment can be described as a set of various actors, connections between them and a list of rules that govern their life processes. Thus, at the present stage of its development, the concept of "Smart Cities" is no longer limited to the use of information technologies, but more often includes an orientation towards improving the quality of human life [13, 14]. Despite some subjectivity of this concept, it should be noted that representatives of different categories of citizens have different expectations from the functionality of objects of the urban environment, thereby forming different, in most cases, contradictory requirements.

Thus, the effectiveness of implementation and social sustainability of certain "smart" solutions can vary significantly depending on the prevailing situation. In this sense, the strategy for the formation of a smart urban environment itself should be flexible and adaptive; both when it is scaled to different real conditions of cities, and take into account the non-stationarity of the conditions of each individual urban environment. Such strategy should be based on some objectively formulated criterion for the effectiveness of the transformation of the environment. The main idea of developing such criterion is to consider the transformation of the urban environment through the prism of changes in the functional state of its objects when using a human-oriented paradigm. That is, the choice of the functional qualities of the objects of the urban environment is determined by the requirements of the townspeople in accordance with the parameters of scenarios of such interactions. These qualities should be critical and sufficient from the point of view of assessing the social sustainability of the urban environment in general and this objects of the urban environment in particular. It should be noted that the presence of a certain quantitative feature that assesses the quality of the functioning of objects of the urban environment objects is a fundamental point. For example, the introduction of digital platforms in the service sector leads, first, to a reduction in the average time for servicing a request in the objects of the urban environment. Thus, the time required to service citizens' requests can be one of the indicators of the effectiveness of the implementation of digital transformation activities. The second example is, for example, the share of citizens expressing a positive attitude towards the activities of the objects of the urban environment. In a narrow sense, the task of transforming the entire urban environment is associated with a change in the functional states of objects of the urban environment, which is expressed in changes in the values of their quantitative indicators of the quality of functioning.

As a first approximation, we can consider functional features that form a certain countable space. Such space is given by the basis $e^{T}=\left(e_{1}, \ldots, e_{p}\right)$, determining the directions of the number axes along which the quantitative functional features of the objects of the urban environment are measured. 
When transforming objects of the urban environment, the following changes are possible:

1) each point in space and / or the center of mass of the system of all points practically do not change their position;

2) some of the points are significantly displaced relative to the previous state, some of the points do not change their position. The center of mass shifts slightly.

3) several comparable parts are displaced in different directions, while maintaining the position of the center of mass;

4) almost all points move in approximately the same direction. The "center of mass" is noticeably shifting.

From general considerations, it follows that option No. 4 and partly No. 2 may reflect the case of an effective transformation of the urban environment. Accordingly, the problem arises of determining a quantitative criterion for the effectiveness of transformation. The idea here is to introduce the metric of such a space and to set criteria for the effectiveness of the implemented solutions by evaluating the average displacements of points in space.

One of the primary indicators of efficiency in this case may be the displacement of the "center of mass" of the system. However, one should also take into account the relative change in each object of the urban environment. The most difficult and least formalized in the task of constructing a criterion is to determine the measure of the distance between objects or the metric of the feature space. It is likely that when selecting the features of the functionality of objects of the urban environment, some of them may be dependent. In addition, signs can be of different types - continuous numeric (for example, waiting time or processing a request), ordinal categorical (scales). In this case, some scaling of the projection on the axis of such features is required. In case of dependent components $e 1, e 2$, ..., e3 vector of features and their different significance in the performance characteristic should probably use the generalized (weighted) Mach-Lanobis distance:

$$
d(x, y)=\|X-Y\|=\sqrt{(X-Y)^{T} \Lambda^{T} \Sigma^{-1} \Lambda(X-Y)},
$$

where $X, Y$ - past and transformed state of the urban environment (see figure 1);

$\Sigma$ - population covariance matrix from which to extract feature values;

$\Lambda$ - some symmetric non-negative definite matrix of "weight" coefficients, which is most often chosen as diagonal.

According to this metric, the average displacement $d_{l}$ can be determined as "centers" of the group of points before and after transformation. It is also necessary to take into account the "sizes" of each of the groups of signs of objects of the urban environment before and after transformation. For this, it is proposed to consider the intragroup variance of a group of points before the transformation $d^{2}\left(x, c_{x}\right)$, where $c_{x}$ - point group center.

The dimensionless quantity $(x, y)=d_{l}^{2} / d^{2}\left(x, c_{x}\right)$, will play the role of the desired criterion, which, on the one hand, depends on the magnitude of the displacement in the feature space during the transformation of the urban environment. With its help, it becomes possible to compare the effectiveness of the implementation of smart solutions and changes in the functionality of objects of the urban environment in relation to the previous state.

This criterion can be used not only to assess the effectiveness of the implemented smart solutions or the social sustainability of the chosen transformation vector, but also when searching for ways to develop the urban environment in a changing climate and the implementation of the Smart Sustainable City (SSC) paradigm [6]. For example, the collection of objects of the urban environment can be considered as objects of generation and consumption of energy. One of the signs that form the eT feature space can be monetary means of payment for consumed energy, etc. With global climate change, local mesoscale climatic conditions of the urban environment can change, leading to an increase 
or decrease in the attractiveness of using local renewable energy sources. In modern concepts, the synthesis of "smart cities" with the system of urban planning can ensure the implementation of the SSC concept. Fundamental importance for the implementation of such a synthesis is the fundamental transition from the technology-oriented to the humanoriented concept of "smart cities" [15], which involves the development of a set of measures and appropriate tools for maintaining, improving and ensuring sustainable development of a comfortable urban environment integrated with natural environment and flexible in relation to possible climatic changes.

\section{$3 \quad$ Models and methods for analyzing the information agenda dynamics on digital transformation of the urban environment}

The digital transformation of the urban environment requires effective feedback from society, which can be obtained when analyzing the information agenda of social networks. Human behavior is highly dependent on the susceptibility of each particular individual and the activity of the social networks that connect them. Long before the spread of online social networks, personal (offline) social interaction (communication) was recognized as the main factor determining the movement of society towards consensus in accepting any dominant ideologies, traditions and individual views. It is based on the analysis of such offline interactions; the dynamics of social influence was thoroughly studied by researchers from various fields of science - sociology, computer science and even physics. In a sociological context, recognition was given to works on the topic of "diffusion of innovations," in which individuals accept new states in behavior, opinion or consumption under the influence of neighboring individuals. Frequently used models for this process are the "threshold" model and the Bass model. A key feature of both of these models is that after an individual accepts a new state; his state remains unchanged in all subsequent periods. Although these models are suitable for analyzing the dynamics of the diffusion of innovations, when investments in a new idea are expensive, they are less suitable for studying the dynamics of competing opinions when the transition from one state to another does not require large "costs".

In most cases, the opinions expressed on social networks about the digital transformation of the urban environment are one of the most pronounced patterns: optimistic and skeptical. The task of effective implementation of smart urban environment technologies in society implies the need for a systematic transformation of skeptical opinions into optimistic ones. For a coordinated effective implementation of these measures, it is advisable to use a powerful mathematical apparatus described in the literature as a model of binary agreement. Consider the dynamics of the evolution of opinions in a binary model. At the initial moment of time, all agents accept a given opinion B, with the exception of a certain proportion of agents $p$, who are "convinced" carriers of opinion A. The concept of convinced agents was introduced earlier in the work and are defined as nodes of a social network that can nodes in order to change their state according to the usual prescribed rules, but which in themselves have a kind of "immunity" to outside influence. In the presence of "convinced" agents who are in state A, the only integral state of the entire social system under consideration is the state of consensus, in which all influencing nodes accept opinion A - the opinion of convinced agents. However, the results of recent studies indicate that values of the fraction $\mathrm{p}$, a consensus can be reached even if the residual number of agents still have competing opinion $\mathrm{B}$. To compose a binary model, we will use the results of work, where a social network of agents is considered, which is a complete graph with the difference $\mathrm{N} \rightarrow \infty$.

Let us define for such a network the density of nodes in the state A, B, as variables $\mathrm{n}_{\mathrm{A}}$, $\mathrm{n}_{\mathrm{B}}$ respectively. Density of nodes in a mixed state $\mathrm{AB}$ denote as $n_{A B}=1-p-n_{A}-n_{B}$. In this 
formula $\mathrm{p}$ - the proportion of the total number of nodes associated with "convinced" agents of the social network, not amenable to changing their minds $A$. Neglecting the correlation between nodes and possible fluctuations, we write down a system of differential equations describing the dynamics of opinions in such a network:

$$
\begin{gathered}
\frac{d n_{A}}{d t}=-n_{A} n_{B}+n_{A B}^{2}+n_{A B} n_{A}+\frac{3}{2} p n_{A B} \\
\frac{d n_{B}}{d t}=-n_{A} n_{B}+n_{A B}^{2}+n_{A B} n_{B}-p n_{B}
\end{gathered}
$$

The values described in equation (1) were obtained by recording all interactions that increase or decrease the density of agents of a certain state (opinion), and to calculate the probability of this interaction occurring. An analysis of the point of reaching consensus on these equations shows that for most values of the fraction $p$, the state of consensus necessarily occurs $(n A=1-p, n B=0)$. This state of consensus is sustainable. However, there is a range of values $\mathrm{p}$, under which it is possible to reach consensus in the public network and subject to $n B>0$, that is, maintaining the share of agents holding an alternative opinion $B$. For a more detailed an

alysis of the dynamics of changes in public opinion, it is proposed to implement procedures for neural network identification of the tonality of citizens' opinions on the digital transformation of the urban environment. Neural networks have proven to be an effective tool for solving problems of analysis and clustering of textual information. In particular, to obtain reliable assessments of public opinion, expressed through the formation of comments in electronic services on the implementation of innovative solutions in the urban environment, it is proposed to use a recurrent neural network (RNN) with a long short-term memory (Long short -term memory - LSTM) [16]. The use of a neural network of this type is due to the increased quality of its characteristics associated with text and speech recognition compared to a number of other alternatives, in particular, multilayer perceptrons [17]. The neural network was trained based on comments published on popular Internet sites for posting user reviews about goods, products and services. The size of the training sample was about two million records. Immediately before training the neural network, a binary classification of the initial data was carried out according to the following principle: when the user rated one, two and three stars, the review was considered "negative", and in the case of four and five stars - "positive". To train the neural network, we used the open software library of machine learning - TensorFlow. This tool is the development of Google. In terms of its functionality, TensorFlow is a powerful tool with wide functional capabilities, but effective interaction with the system requires a highly qualified programmer. In view of this, to work with this tool, the Keras library was used, which is an add-on over the TensorFlow library and allows to significantly simplifying the work associated with the formation of a neural network [18]. The software implementation of the module was written in the high-level Python programming language using a free cloud service for artificial intelligence developers Google Colab. This service allows you to develop applications for training multilayer neural networks from a browser, providing the computing power of the powerful NVIDIA Tesla K80 graphics processor with $13 \mathrm{~GB}$ of video memory. The presence of a GPU accelerator is a critical factor for increasing the learning rate of deep neural network models, without which it is almost impossible to fully experiment with the structure of a neural network. Default Colaboratory не использует ускорителей вычислений GPU или TPU. You can connect them in the Runtime menu -> Change runtime type -> Hardware ac-celerator. In the list that appears, select "GPU" or "TPU" After selecting the type of accelerator, the virtual machine to which the Colaboratory laptop is connected will restart and the selected mode will become available.

The advantages of this service are also the fact that most of the libraries necessary for the work are preinstalled. At the same time, if necessary, the ability to install the required 
package is also supported. Another important advantage is the ability to carry out joint work in the Colaboratory on writing and testing the program year. To train the neural network, we used the open software library of machine learning - TensorFlow. This tool is the development of Google and has an open source, which allows you to flexibly embed it to solve various problems. In terms of its functionality, TensorFlow is a powerful tool with wide functional capabilities, but effective interaction with the system requires a highly qualified programmer. In view of this, to work with this tool, the Keras library was used, which is an add-on over the TensorFlow library and allows to significantly simplifying the procedures for forming a neural network.

\section{Conclusions}

The processes of transition from a technology-oriented paradigm of the formation of a smart urban environment to a person-centered concept, taking into account the individual needs of individual citizens and various social groups, requires the creation of developed information technologies for the verification of measures for the digital transformation of the urban environment, based on the use of the opinions of citizens, assessed using a recurrent neural network using the TensorFlow framework. The approach described in the article is focused on providing decision-makers with the necessary information support, which allows them to form reasoned, balanced decisions on the development of urban areas.

Thus, the main direction of development of the proposed approach is the synthesis of the methodology for assessing the level of comfort of the urban environment. This is a complex, interdisciplinary scientific study that will require the collaborative work of experts from various subject areas: urbanists, sociologists, specialists in simulation modeling, GIS technologies and end-to-end digital technologies. This methodology will make it possible to analyze the risks associated with the unsystematic, excessive introduction of technical innovations into the lives of citizens, disrupting the usual processes of their life, as well as to implement an adequate digital model of the city, taking into account the principles of sustainable development of urban space, which will become a basic tool for the formation of strategies. development of the urbanized environment.

An example of using the proposed approach for analyzing various options for digital transformation of the urban environment in various information situations is given. The proposed approach allows, among other things, to generate scatter diagrams, which are a powerful visualization tool in assessing various strategies for digital transformation of the urban environment.

\section{Acknowledgements}

The study was carried out with partial financial support from the Russian Foundation for Basic Research and the Government of Sevastopol in the framework of the scientific project № 20-47-920006, as well as the Sevastopol State University in the framework of the internal grant № 35/06-31 under the project "Application of big data technologies for solving problems of environmental monitoring".

\section{References}

1. The UNECE-ITU Smart Sustainable Cities Indicators, Economic and Social Council United Nations, http://www.unece.org/

2. V. Albino, U. Berardi, R.M. Dangelico, J. Urban Technol, 22(1), 3 (2015) 
3. M. Hara, et al., Sustainability, 8(3), 206 (2016)

4. S. E. Bibri, Sustainable Cities Soc., 38, 758 (2018)

5. P. Chamoso, et al, Wireless Commun. Mobile Comput (2018)

6. M. Höjer, J. Wangel, Smart Sustainable Cities: Definition and Challenges, 333 (2015)

7. C. J. Martin, J. Evans, A. Karvonen, Technol. Forecasting Social Change, 133, 269 (2018)

8. S. Al-Nasrawi, C. Adams, A. El-Zaart, J. Inf. Syst. Technol. Manage, 12(3), 541 (2015)

9. E. Park, A.P. del Pobil, S.J. Kwon, Sustainability, 10, 1388 (2018)

10. M. Freudendal-Pedersen, S. Kesselring, E. Servou, Sustainability, 11(1), 1 (2019)

11. V. Albino, U. Berardi, R.M. Dangelico, J. Urban Technol, 22(1), 3 (2015)

12. M. Batty, K.W. Axhausen, F. Giannotti, A. Pozdnoukhov, A. Bazzani, M. Wachowicz, G. Ouzounis, Y. Portugali, Eur. Phys. J. Spec. Top., 214(1), 481 (2012)

13. R.K.R. Kummitha, N. Crutzen, Cities, 67, 43 (2017)

14. H. Sak, A. Senior, F. Beaufays, Proceedings of the Annual Conference of the International Speech Communication Association, 338 (2014)

15. Li Xiangang, Wu Xihong, IEEE International Conference on Acoustics, Speech and Signal Processing (ICASSP), 4520 (2015)

16. H.J. Eyre, D.P. Lange, Hands-On Machine Learning with Scikit-Learn, Keras, and TensorFlow: Concepts, Tools, and Techniques to Build Intelligent Systems, 817 (2019)

17. R. Betancourt, S. Chen, Pandas Library. In: Python for SAS Users, 452 (2019)

18. H. Sak, A. Senior, F. Beaufays, Proceedings of the Annual Conference of the International Speech Communication Association, 338 (2014) 